\title{
Towards Resource Theory of Coherence in Distributed Scenarios
}

\author{
Alexander Streltsov, ${ }^{1,2,3,4, *}$ Swapan Rana, ${ }^{4}$ Manabendra Nath Bera, ${ }^{4}$ and Maciej Lewenstein ${ }^{4,5}$ \\ ${ }^{1}$ Faculty of Applied Physics and Mathematics, Gdańsk University of Technology, 80-233 Gdańsk, Poland \\ ${ }^{2}$ National Quantum Information Center in Gdańsk, 81-824 Sopot, Poland \\ ${ }^{3}$ Dahlem Center for Complex Quantum Systems, Freie Universität Berlin, D-14195 Berlin, Germany \\ ${ }^{4}$ ICFO_Institut de Ciències Fotòniques, The Barcelona Institute of Science and Technology, \\ 08860 Castelldefels, Spain \\ ${ }^{5}$ ICREA, Lluis Companys 23, 08010 Barcelona, Spain \\ (Received 29 September 2015; revised manuscript received 21 December 2016; published 6 March 2017)
}

\begin{abstract}
The search for a simple description of fundamental physical processes is an important part of quantum theory. One example for such an abstraction can be found in the distance lab paradigm: if two separated parties are connected via a classical channel, it is notoriously difficult to characterize all possible operations these parties can perform. This class of operations is widely known as local operations and classical communication. Surprisingly, the situation becomes comparably simple if the more general class of separable operations is considered, a finding that has been extensively used in quantum information theory for many years. Here, we propose a related approach for the resource theory of quantum coherence, where two distant parties can perform only measurements that do not create coherence and can communicate their outcomes via a classical channel. We call this class local incoherent operations and classical communication. While the characterization of this class is also difficult in general, we show that the larger class of separable incoherent operations has a simple mathematical form, yet still preserves the main features of local incoherent operations and classical communication. We demonstrate the relevance of our approach by applying it to three different tasks: assisted coherence distillation, quantum teleportation, and single-shot quantum state merging. We expect that the results we obtain in this work also transfer to other concepts of coherence that are discussed in recent literature. The approach we present here opens new ways to study the resource theory of coherence in distributed scenarios.
\end{abstract}

DOI: 10.1103/PhysRevX.7.011024

\section{INTRODUCTION}

The resource theory of quantum coherence is a vivid research topic, and various approaches in this direction have been presented over the past few years [1-6]. The formalism proposed recently by Baumgratz et al. [5] has triggered the interest of several authors, and a variety of results have been obtained since then. One line of research is the formulation and interpretation of new coherence quantifiers [7-11], in particular, those arising from quantum correlations such as entanglement $[12,13]$. The study of coherence dynamics under noisy evolution is another promising research direction [14-18]. The role of coherence in biological systems [19-21], thermodynamics [22-26], spin models [27-29], and other related tasks in quantum theory [30-38] has also been investigated.

In the framework introduced by Baumgratz et al. [5], quantum states that are diagonal in some fixed basis $\{|i\rangle\}$

\footnotetext{
*streltsov.physics@gmail.com
}

Published by the American Physical Society under the terms of the Creative Commons Attribution 4.0 International license. Further distribution of this work must maintain attribution to the author(s) and the published article's title, journal citation, and DOI.
Subject Areas: Quantum Physics

are called incoherent: these are all states of the form $\rho=\sum_{k} p_{k}|k\rangle\langle k|$. A quantum operation is called incoherent if it can be written in the form $\Lambda(\rho)=\sum_{l} K_{l} \rho K_{l}^{\dagger}$, with incoherent Kraus operators $K_{l}$, i.e., $K_{l}|m\rangle \sim|n\rangle$, where $|m\rangle$ and $|n\rangle$ are elements of the incoherent basis. Significant progress within this resource theory has been achieved by Winter and Yang [39]. In particular, they introduced the distillable coherence and presented a closed formula for it for all quantum states. Similar to the distillable entanglement [40,41], the distillable coherence is defined as the maximal rate for extracting maximally coherent singlequbit states,

$$
\left|\Psi_{2}\right\rangle=\frac{1}{\sqrt{2}}(|0\rangle+|1\rangle),
$$

from a given mixed state $\rho$ via incoherent operations. Another closely related quantity is the relative entropy of coherence, initially defined as [5]

$$
C_{r}(\rho)=\min _{\sigma \in \mathcal{I}} S(\rho \| \sigma),
$$

where $S(\rho \| \sigma)=\operatorname{Tr}\left[\rho \log _{2} \rho\right]-\operatorname{Tr}\left[\rho \log _{2} \sigma\right]$ is the relative entropy, and the minimum is taken over the set of incoherent states $\mathcal{I}$. Crucially, the relative entropy of 
coherence is equal to the distillable coherence and can be evaluated exactly $[5,39]$ :

$$
C_{d}(\rho)=C_{r}(\rho)=S(\Delta(\rho))-S(\rho),
$$

where $S(\rho)=-\operatorname{Tr}\left[\rho \log _{2} \rho\right]$ is the von Neumann entropy and $\Delta(\rho)=\sum_{k}\langle k|\rho| k\rangle|k\rangle\langle k|$ denotes dephasing of $\rho$ in the incoherent basis.

Recently, various alternative concepts of coherence have been presented in the literature. We briefly review the most important approaches in the following, and refer to Refs. [6,42-44] and references therein for more details. While all these notions agree on the definition of incoherent states as states that are diagonal in a fixed reference basis, they differ significantly in the definition of the corresponding free operations. A notable approach in this context is the notion of translationally invariant operations; these are operations that commute with unitary translations $e^{-i H t}$ for some Hamiltonian $H[2,3]$. As was shown by Marvian et al. [45], for nondegenerate Hamiltonians translationally invariant operations are a proper subset of incoherent operations. Moreover, translationally invariant operations have several desirable properties, such as a free dilation: they can be implemented by introducing an incoherent ancilla, performing a global incoherent unitary followed by an incoherent measurement on the ancilla, and postselection on the outcomes [43]. As was also shown in Ref. [43], incoherent operations introduced by Baumgratz et al. [5] in general do not have such a free dilation. While the existence of a free dilation is clearly appealing from the resourcetheoretic perspective, the question of whether every reasonable resource theory should have a free dilation is still not fully settled.

By a similar motivation, Chitambar and Gour [42] introduced the concept of physical incoherent operations. These operations have a free dilation if one allows incoherent projective measurements on the ancilla followed by classical processing of the outcomes. Interestingly, the resource theory obtained in this way does not have a maximally coherent state; i.e., there is no unique state from which all other states can be obtained via physically incoherent operations. This is also true for genuinely incoherent and fully incoherent operations [44]. Genuinely incoherent operations are defined as operations that preserve all incoherent states; they capture the framework of coherence under additional constrains such as energy preservation [44]. Moreover, all genuinely incoherent operations are incoherent regardless of a particular experimental realization. Fully incoherent operations is the most general set having this property [44].

An alternative approach to coherence was made by Yadin et al. [46], who studied quantum processes that do not use coherence. Such a process cannot be used to detect coherence in a quantum state; i.e., an experimenter who has access to those operations and incoherent von Neumann measurements will not be able to decide if a quantum state has coherence or not. These operations coincide with strictly incoherent operations, which were introduced earlier by Winter and Yang [39].

As has been shown in several recent works, quantum coherence plays an important role in various tasks that are based on the laws of quantum mechanics. One such task is quantum state merging, which was first introduced and studied in Reffs. [47,48]. The interplay between entanglement and local coherence in this task was investigated very recently in Ref. [49]. An important concept in this context is the notion of local quantum-incoherent operations and classical communication (LQICC) [50]. This class of operations is similar to the class of local operations and classical communication (LOCC) where Alice and Bob can apply local measurements and share their outcomes via a classical channel, with the only difference that Bob's measurements have to be incoherent [50].

In this paper, we consider the situation where both parties, Alice and Bob, can perform only incoherent measurement on their parts. The corresponding class is called local incoherent operations and classical communication (LICC). Moreover, we also generalize these notions to separable operations known from entanglement theory [41,51,52], thus introducing separable incoherent (SI) operations, and separable quantum-incoherent (SQI) operations. We study the relation of all these classes among each other, and apply them to the task of assisted coherence distillation, which was first introduced in Ref. [50]. We also introduce and discuss the task of incoherent teleportation and study the relation between our classes on single-shot quantum state merging. As we discuss in Sec. VI, we expect that the ideas we present in this work will find applications beyond quantum information theory, most prominently in quantum thermodynamics and related research areas.

\section{CLASSES OF INCOHERENT OPERATIONS IN DISTRIBUTED SCENARIOS}

The framework of local operations and classical communication is one of the most important concepts in entanglement theory, as it describes all transformations that two separated parties (Alice and Bob) can perform if they apply local quantum measurements and have access to a classical channel $[41,53]$. These operations are difficult to capture mathematically, since a general LOCC operation can involve an arbitrary number of rounds of classical communication [41,53]. However, in many relevant cases it is enough to consider the informal definition given above. In a similar fashion, we define the class of local incoherent operations and classical communication: these are LOCC operations with the additional constraint that the local measurements of Alice and Bob have to be incoherent. We also consider the case where Alice can perform arbitrary quantum measurements, while Bob is restricted to incoherent measurements only. The corresponding class of 
operations is called local quantum-incoherent operations and classical communication [50].

Another important framework in entanglement theory is separable operations. While this class is larger than LOCC [54], it has a simple mathematical description, and still preserves the main features of LOCC [41,53]. Separable operations were initially introduced in Refs. [51,52] as follows:

$$
\Lambda_{\mathrm{S}}\left[\rho^{A B}\right]=\sum_{i} A_{i} \otimes B_{i} \rho^{A B} A_{i}^{\dagger} \otimes B_{i}^{\dagger} .
$$

The product operators $A_{i} \otimes B_{i}$ are Kraus operators; i.e., they fulfill the completeness condition

$$
\sum_{i} A_{i}^{\dagger} A_{i} \otimes B_{i}^{\dagger} B_{i}=\mathbb{1} .
$$

The set of all separable operations is called S. If all the operators $A_{i}$ and $B_{i}$ are incoherent, i.e., if they satisfy the conditions

$$
\begin{aligned}
A_{i}|k\rangle^{A} & \sim|l\rangle^{A}, \\
B_{i}|m\rangle^{B} & \sim|n\rangle^{B},
\end{aligned}
$$

we call the total operation separable incoherent [55]. In the more general case where only Bob's operators $B_{i}$ are incoherent-and, thus, only Eq. (7) is satisfied-the corresponding operation is called separable quantum incoherent.

Having introduced the notion of LICC, LQICC, SI, and SQI operations, we now study the action of these operations on the initial incoherent state $|0\rangle^{A}|0\rangle^{B}$. It is easy to see that the set of states created from $|0\rangle^{A}|0\rangle^{B}$ via LICC and via SI operations is the same, and given by states of the form

$$
\rho_{\mathrm{i}}=\sum_{k, l} p_{k l}|k\rangle\left\langle\left. k\right|^{A} \otimes \mid l\right\rangle\left\langle\left. l\right|^{B} .\right.
$$

States of this form are known as fully incoherent states $[12,14]$, and the set of all such states is denoted by $\mathcal{I}$. Similarly, the set of states created from $|0\rangle^{A}|0\rangle^{B}$ via LQICC and via SQI operations is the set of quantum-incoherent states $\mathcal{Q I}$. These are states of the form [50]

$$
\rho_{\mathcal{Q I}}=\sum_{j} p_{j} \sigma_{j}^{A} \otimes|j\rangle\left\langle\left. j\right|^{B} .\right.
$$

From the above results, we immediately see that SI operations map the set of fully incoherent states $\mathcal{I}$ onto itself, and the same is true for LICC operations. Similarly, SQI operations and LQICC operations map the set of fully incoherent states $\mathcal{I}$ onto the larger set of quantumincoherent states $\mathcal{Q I}$. These statements are summarized in the following equalities:

$$
\begin{gathered}
\Lambda_{\mathrm{SI}}[\mathcal{I}]=\Lambda_{\mathrm{LICC}}[\mathcal{I}]=\mathcal{I}, \\
\Lambda_{\mathrm{SQI}}[\mathcal{I}]=\Lambda_{\mathrm{LQICC}}[\mathcal{I}]=\mathcal{Q I}
\end{gathered}
$$

In general, LICC is the weakest set of operations, and the set of separable operations $S$ is the most powerful set of operations considered here. Thus, we get the following inclusions:

$$
\begin{aligned}
& \mathrm{LICC} \subset \mathrm{SI} \subset \mathrm{SQI} \subset \mathrm{S}, \\
& \mathrm{LICC} \subset \mathrm{LQICC} \subset \mathrm{SQI} \subset \mathrm{S}, \\
& \mathrm{LICC} \subset \mathrm{LQICC} \subset \mathrm{LOCC} \subset \mathrm{S} .
\end{aligned}
$$

For all of the above inclusions it is straightforward to see the weaker form $X \subseteq Y$, where $X$ and $Y$ is the corresponding set of operations. For most of these inclusions, $X \subset Y$ can then be proven by applying the corresponding sets of operations to the set of fully incoherent states $\mathcal{I}$. As an example, SI $\subset$ SQI follows from the fact that $\Lambda_{\mathrm{SI}}[\mathcal{I}]=\mathcal{I}$ while $\Lambda_{\mathrm{SQI}}[\mathcal{I}]=\mathcal{Q I}$. The same arguments apply to all the above inclusions apart from

$$
\begin{gathered}
\mathrm{LICC} \subset \mathrm{SI}, \\
\mathrm{LQICC} \subset \mathrm{SQI},
\end{gathered}
$$

and LOCC $\subset \mathrm{S}$. As already noted above Eq. (4), the inclusion LOCC $\subset \mathrm{S}$ was proven by Bennett et al. [54], and the remaining two can be proven using very similar arguments. In particular, Bennett et al. [54] presented a separable operation that cannot be implemented via LOCC. The corresponding product operators of this operation have the following form [see Eq. (4) in Ref. [54]]:

$$
A_{i} \otimes B_{i}=|i\rangle\left\langle\left.\alpha_{i}\right|^{A} \otimes \mid i\right\rangle\left\langle\left.\beta_{i}\right|^{B} .\right.
$$

The particular expressions for $\left|\alpha_{i}\right\rangle$ and $\left|\beta_{i}\right\rangle$ were given in Ref. [54] (see also the Appendix), but are not important for the rest of our proof. However, it is important to note that the states $|i\rangle^{A}$ and $|i\rangle^{B}$ are incoherent states of Alice and Bob, respectively. It is straightforward to see that this separable operation is also a SI operation. Moreover, since this operation cannot be implemented via LOCC, it also cannot be implemented via LICC. This completes the proof of Eq. (13). The proof of Eq. (14) follows by the same reasoning.

The hierarchy of the sets LICC, SI, LOCC, and S is shown in Fig. 1. Note that the above reasoning also implies that the sets LOCC and SI have an overlap, but one is not a subset of the other. Moreover, the figure also depicts a region of operations (crossed area) that are simultaneously contained in LOCC and SI, but not in LICC. Such 


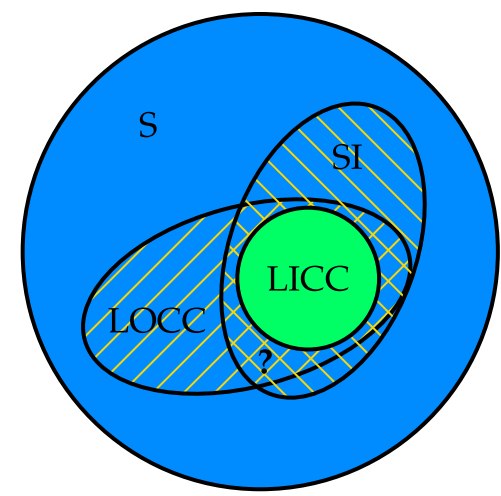

FIG. 1. Hierarchy of LICC, SI, LOCC, and separable operations S. The set of LICC operations is the weakest set, and $\mathrm{S}$ is the most powerful set. The crossed region displays operations that are in LOCC and SI, but not in LICC. It remains open if such operations exist. For simplicity, we do not display the sets LQICC and SQI.

operations would have the property that they cannot create any bipartite coherence. On the other hand, they can be implemented via local operations and classical communication, but for that they require local coherent operations on at least one of the parties. It remains an interesting open question as to whether such operations exist at all. If the answer to this question is negative, the intersection of LOCC and SI is equal to LICC. We also mention that similar questions arise if we consider the sets LQICC and SQI. These sets are not included in Fig. 1 for simplicity. Their inclusions are shown in Eq. (12). In the following section, we apply the tools presented here to the task of assisted coherence distillation, initially presented in Ref. [50].

\section{ASSISTED COHERENCE DISTILLATION}

\section{A. General setting}

The task of assisted coherence distillation via bipartite LQICC operations was introduced and studied in Ref. [50]. In this task, Alice and Bob share many copies of a given state $\rho=\rho^{A B}$. The aim of the process is to asymptotically distill maximally coherent single-qubit states on Bob's side. In particular, we are interested in the maximal possible rate for this procedure.

In the following, we extend this notion beyond LQICC operations. For this we consider the maximal amount of coherence that can be distilled on Bob's side via the set of operations $X$, where $X$ is either LICC, LQICC, SI, or SQI [56]. The corresponding distillable coherence on Bob's side is denoted by $C_{X}$ and can, in general, be given as follows:

$$
C_{X}^{A \mid B}(\rho)=\sup \left\{c: \lim _{n \rightarrow \infty}\left(\inf _{\Lambda \in X} \| \operatorname{Tr}_{A}\left[\Lambda\left[\rho^{\otimes n}\right]\right]-|c\rangle\left\langle\left. c\right|^{\otimes n} \|\right)=0\right\},\right.
$$

where $|c\rangle=|c\rangle^{B}$ is a state on Bob's subsystem with distillable coherence $C_{d}(|c\rangle)=c$ and $\|M\|=\operatorname{Tr}\left[\sqrt{M^{\dagger} M}\right]$ is the trace norm. For more details regarding this definition of $C_{X}$ and for equivalent expressions, we refer the reader to Sec. III D. The quantity $C_{\mathrm{LQICC}}^{A \mid B}$ was introduced in Ref. [50], where it was called distillable coherence of collaboration.

From Eqs. (12a) and (12b) we immediately see that all quantities $C_{X}$ considered here are between $C_{\mathrm{LICC}}$ and $C_{\mathrm{SQI}}$. In the following, we also consider the QI relative entropy, which was defined in Ref. [50] as follows:

$$
C_{r}^{A \mid B}(\rho)=\min _{\sigma \in \mathcal{Q} I} S(\rho \| \sigma) .
$$

As was also shown in Ref. [50], the QI relative entropy can be written in closed form:

$$
C_{r}^{A \mid B}(\rho)=S\left(\Delta^{B}(\rho)\right)-S(\rho) .
$$

Note that the QI relative entropy is additive and does not increase under SQI operations. Thus, it does not increase under any set of operations $X$ considered here.

It is interesting to compare the QI relative entropy to the basis-dependent quantum discord, which was initially introduced in Ref. [57] and can be written as

$$
\delta^{A \mid B}(\rho)=I^{A: B}(\rho)-I^{A: B}\left(\Delta^{B}[\rho]\right),
$$

with the mutual information $I^{A: B}(\rho)=S\left(\rho^{A}\right)+S\left(\rho^{B}\right)-$ $S\left(\rho^{A B}\right)$. Recently, Yadin et al. [46] studied the role of this quantity within the resource theory of coherence. Contrary to the results presented in Ref. [57], the basis-dependent discord vanishes on a larger set of states than the QI relative entropy. While the latter is zero if and only if the corresponding state is quantum incoherent, the basisdependent discord vanishes for all states of the form $\rho=\sum_{i} p_{i} \rho_{i}^{A} \otimes \rho_{i}^{B}$, where the states $\rho_{i}^{B}$ are perfectly distinguishable by measurements in the incoherent basis [46]. This is, in particular, the case if $\rho$ is quantum incoherent or a product state, and other examples have also been presented in Ref. [46].

Quite remarkably, we see below that $C_{\mathrm{SI}}$ is equal to $C_{\mathrm{SQI}}$ for all states $\rho$, and, moreover, all quantities $C_{X}$ are bounded above by the QI relative entropy. The following inequality summarizes these results:

$$
C_{\mathrm{LICC}}^{A \mid B} \leq C_{\mathrm{LQICC}}^{A \mid B} \leq C_{\mathrm{SI}}^{A \mid B}=C_{\mathrm{SQI}}^{A \mid B} \leq C_{r}^{A \mid B} .
$$

The equality $C_{\mathrm{SI}}^{A \mid B}=C_{\mathrm{SQI}}^{A \mid B}$ is proven in the following proposition, and the bound $C_{\mathrm{SQI}}^{A \mid B} \leq C_{r}^{A \mid B}$ is proven in Theorem 2.

Proposition 1.-For an arbitrary bipartite state $\rho=\rho^{A B}$, the following equality holds: 


$$
C_{\mathrm{SI}}^{A \mid B}(\rho)=C_{\mathrm{SQI}}^{A \mid B}(\rho)
$$

Proof.-In the first step of the proof we show that for any state $\rho^{A B}$ and an arbitrary SQI operation $\Lambda_{\mathrm{SQI}}$ there exists a SI operation $\Lambda_{\mathrm{SI}}$ leading to the same reduced state of Bob:

$$
\operatorname{Tr}_{A}\left[\Lambda_{\mathrm{SI}}\left[\rho^{A B}\right]\right]=\operatorname{Tr}_{A}\left[\Lambda_{\mathrm{SQI}}\left[\rho^{A B}\right]\right]
$$

The desired SI operation is given by

$$
\Lambda_{\mathrm{SI}}\left[\rho^{A B}\right]=\sum_{i} \Pi_{i}^{A} \Lambda_{\mathrm{SQI}}\left[\rho^{A B}\right] \Pi_{i}^{A}
$$

where $\Pi_{i}^{A}=|i\rangle\left\langle\left. i\right|^{A}\right.$ is a complete set of orthogonal projectors onto the incoherent basis of Alice. It is straightforward to see that the above operation satisfies Eq. (22) and is indeed separable and incoherent.

Now, given a state $\rho=\rho^{A B}$ with $C_{\mathrm{SQI}}^{A \mid B}(\rho)=c$, for any $\varepsilon>0$, there exists an integer $n \geq 1$ and a SQI operation $\Lambda_{\mathrm{SQI}}$ acting on $n$ copies of $\rho$ such that

$$
\| \operatorname{Tr}_{A}\left[\Lambda_{\mathrm{SQI}}\left[\rho^{\otimes n}\right]\right]-|c\rangle\left\langle\left. c\right|^{\otimes n} \| \leq \varepsilon\right.
$$

where $|c\rangle$ is a pure state with $C_{d}(|c\rangle)=c$. By using Eq. (22), it follows that for any $\varepsilon>0$ and some integer $n \geq 1$ there also exists a SI operation $\Lambda_{\mathrm{SI}}$ with the same property:

$$
\| \operatorname{Tr}_{A}\left[\Lambda_{\mathrm{SI}}\left[\rho^{\otimes n}\right]\right]-|c\rangle\left\langle\left. c\right|^{\otimes n} \| \leq \varepsilon .\right.
$$

These arguments show that $C_{\mathrm{SI}}$ is bounded below by $C_{\mathrm{SQI}}$. The proof of the theorem is complete by noting that $C_{\mathrm{SI}}$ is also bounded above by $C_{\mathrm{SQI}}$ since SI $\subset \mathrm{SQI}$.

This proposition shows that SQI operations do not provide an advantage when compared to SI operations in the considered task: both sets of operations lead to the same maximal performance. This result is remarkable since the sets SI and SQI are not equal. It is now tempting to assume that the same method can also be used to prove that $C_{\mathrm{LICC}}^{A \mid B}$ is equal to $C_{\mathrm{LQICC}}^{A \mid B}$, i.e., that quantum operations on Alice's side do not provide any advantage for assisted coherence distillation. Note, however, that the above proof does not apply to this scenario, and thus the question remains open for general mixed states. However, as we see in the next section, for pure states $C_{\mathrm{LICC}}^{A \mid B}$ is indeed equal to $C_{\mathrm{LQICC}}^{A \mid B}$.

In the following theorem, we prove that $C_{\mathrm{SQI}}$ is bounded above by the QI relative entropy. This completes the proof of the inequality Eq. (20).
Theorem 2.-For any bipartite state, $\rho=\rho^{A B}$ holds:

$$
C_{\mathrm{SQI}}^{A \mid B}(\rho) \leq C_{r}^{A \mid B}(\rho)
$$

Proof.-This proof goes along similar lines of reasoning as the proof of Theorem 3 in Ref. [50]. From the definition of $C_{\mathrm{SQI}}$ in Eq. (16), it follows that for any $\varepsilon>0$ there exists a state $|\phi\rangle$, an integer $n>1$, and a SQI protocol $\Lambda_{\mathrm{SQI}}$ acting on $n$ copies of $\rho=\rho^{A B}$ such that

$$
\begin{gathered}
C_{\mathrm{SQI}}^{A \mid B}(\rho)-C_{r}(|\phi\rangle) \leq \varepsilon, \\
\left\|\Lambda_{\mathrm{SQI}}\left[\rho^{\otimes n}\right]-\rho_{f}^{\otimes n}\right\| \leq \varepsilon,
\end{gathered}
$$

with the final state $\rho_{f}=|0\rangle\left\langle\left. 0\right|^{A} \otimes \mid \phi\right\rangle\left\langle\left.\phi\right|^{B}\right.$.

Equation (28) together with the continuity of QI relative entropy [58] implies that for any $0<\varepsilon \leq 1 / 2$ there exists an integer $n \geq 1$ and a SQI protocol $\Lambda_{\mathrm{SQI}}$ acting on $n$ copies of $\rho$, such that

$C_{r}^{A \mid B}\left(\Lambda_{\mathrm{SQI}}\left[\rho^{\otimes n}\right]\right) \geq C_{r}^{A \mid B}\left(\rho_{f}^{\otimes n}\right)-2 n \varepsilon \log _{2} d-2 h(\varepsilon)$,

where $h(x)=-x \log _{2} x-(1-x) \log _{2}(1-x) \quad$ is the binary entropy and $d$ is the dimension of $A B$. Now, we use the fact that the QI relative entropy is additive [50] and does not increase under SQI operations. This means that for any $0<\varepsilon \leq 1 / 2$ there exists an integer $n \geq 1$ such that

$$
C_{r}^{A \mid B}(\rho) \geq C_{r}^{A \mid B}\left(\rho_{f}\right)-2 \varepsilon \log _{2} d-\frac{2}{n} h(\varepsilon) .
$$

The above inequality together with the fact $C_{r}^{A \mid B}\left(\rho_{f}\right)=$ $C_{r}(|\phi\rangle)$ and Eq. (27) implies that for any $0<\varepsilon \leq 1 / 2$ there exists an integer $n \geq 1$ such that

$$
C_{r}^{A \mid B}(\rho) \geq C_{\mathrm{SQI}}^{A \mid B}(\rho)-\varepsilon-2 \varepsilon \log _{2} d-\frac{2}{n} h(\varepsilon) .
$$

This completes the proof of the theorem.

Proposition 1 and Theorem 2 in combination imply Eq. (20). It remains an open question of whether the inequalities in Eq. (20) are strict. As we see in the next section, this is not the case for a pure state: in this case, all quantities are equal to the von Neumann entropy of the fully decohered state of Bob $\Delta\left(\rho^{B}\right)$.

Before we turn our attention to this question, we first characterize all quantum states that are useful for assisted coherence distillation via the sets of operations $X$ considered above. Note that a quantum-incoherent state cannot be used for extraction of coherence on Bob's side via any set of operations $X$. On the other hand, as we show in the following theorem, any state that is not quantum incoherent can be used for extracting coherence via LICC. 
Theorem 3.-A state $\rho=\rho^{A B}$ has $C_{\mathrm{LICC}}^{A \mid B}(\rho)>0$ if and only if it is not quantum incoherent.

Proof.-As $C_{\mathrm{LICC}}^{A \mid B}\left(\rho^{A B}\right)=0$ for any quantum-incoherent state $\rho^{A B}$, the claim follows if we show that $\rho^{A B}$ is not QI implies $C_{\text {LICC }}^{A \mid B}\left(\rho^{A B}\right)>0$. Without loss of generality, let the non-QI state be given as

$$
\rho^{A B}=\sum_{i, j}\left|e_{i}\right\rangle\left\langle\left. e_{j}\right|^{A} \otimes N_{i j}^{B},\right.
$$

where $\left\{\left|e_{i}\right\rangle^{A}\right\}$ is an orthonormal basis for Alice's Hilbert space [59] and $N_{i j}^{B}$ are some operators on Bob's space. By the non-QI assumption, at least one of the $\left\{N_{i j}\right\}$ has offdiagonal elements.

We note that $N_{i i} \geq 0$, so $N_{i i} \neq 0 \Leftrightarrow \operatorname{Tr}\left[N_{i i}\right]>0$. If an $N_{i i}$ has off-diagonal elements, then the state of Bob after Alice's incoherent measurement with Kraus operator $K_{i}^{A}=|i\rangle\left\langle\left. e_{i}\right|^{A}\right.$ is $\rho_{i}^{B} \sim N_{i i}$, with nonzero probability $\operatorname{Tr}\left[N_{i i}\right]>0$. Hence, $C_{\text {LICC }}^{A \mid B}\left(\rho^{A B}\right)>0$.

We now assume that all $N_{i i}$ are diagonal. In this caseby the non-QI assumption-some of the operators $N_{k l}$ must have off-diagonal elements for some $k \neq l$. If one of the operators $N_{k l}$ (by Hermiticity of $\rho$, we can assume $k<l$ without loss of generality) has some off-diagonal elements, then at least one of the operators $P:=N_{k l}+N_{k l}^{\dagger}, Q:=$ $i\left(N_{k l}-N_{k l}^{\dagger}\right)$ will also have off-diagonal elements. Depending on which is the case, Alice performs an incoherent measurement containing the Kraus operator $K_{P}:=|0\rangle\left\langle e_{P}\right|$ or $K_{Q}:=|0\rangle\left\langle e_{Q}\right|$, where we define $\left|e_{P}\right\rangle:=$ $\cos \theta\left|e_{k}\right\rangle+\sin \theta\left|e_{l}\right\rangle,\left|e_{Q}\right\rangle:=\cos \theta\left|e_{k}\right\rangle+i \sin \theta\left|e_{l}\right\rangle$, and the unknown parameter $\theta$ will be determined soon. In the first case, the postmeasurement state of Bob is given by

$\rho_{\theta}^{B} \sim \cos ^{2} \theta N_{k k}+\sin ^{2} \theta N_{l l}+\cos \theta \sin \theta\left(N_{k l}+N_{k l}^{\dagger}\right)$,

which, by assumption, has off-diagonal elements. Note that since $\sin ^{2} \theta, \cos ^{2} \theta, \sin \theta \cos \theta$ are linearly independent functions, there is always some $0<\theta<\pi / 2$ for which the trace of the right-hand side is nonzero; i.e., with nonzero probability $\rho_{\theta}^{B}$ is coherent. Similarly, in the other case, where $i\left(N_{k l}-N_{k l}^{\dagger}\right)$ is assumed to have off-diagonal elements, the postmeasurement state of Bob is coherent with nonzero probability.

Thus, whenever $\rho^{A B}$ is not QI, with nonzero probability Alice can steer Bob's state to a coherent one, which Bob can distill by using the methods presented by Winter and Yang [39], so $C_{\text {LICC }}^{A \mid B}\left(\rho^{A B}\right)>0$.

Since LICC is the weakest set of operations considered here, this theorem also means that a state that is not quantum incoherent can be used for coherence distillation on Bob's side via any set of operations presented above.

\section{B. Pure states}

In the following we study the scenario where the state shared by Alice and Bob is pure, and the aim is to distill coherence at maximal rate on Bob's side via the sets of operations presented above. Before we study this task, we recall the definition of coherence of assistance given in Ref. [50]:

$$
C_{a}(\rho)=\max \sum_{i} p_{i} C_{r}\left(\left|\psi_{i}\right\rangle\right),
$$

where the maximum is performed over all pure state decompositions of $\rho$. We now prove the following lemma.

Lemma 4.-For any pure state $|\Psi\rangle^{A B}$ there exists an incoherent measurement on Alice's side such that

$$
\sum_{i} p_{i} C_{r}\left(\left|\psi_{i}\right\rangle^{B}\right)=C_{a}\left(\rho^{B}\right),
$$

where $\left|\psi_{i}\right\rangle^{B}$ are Bob's postmeasurement states with corresponding probability $p_{i}$.

Proof.-Note that any pure state can be written as

$$
|\Psi\rangle^{A B}=\sum_{i} \sqrt{p_{i}}\left|e_{i}\right\rangle^{A}\left|\psi_{i}\right\rangle^{B}
$$

where the states $\left|e_{i}\right\rangle^{A}$ are mutually orthogonal (but not necessarily incoherent), and the states $\left|\psi_{i}\right\rangle^{B}$ together with probabilities $p_{i}$ fulfill Eq. (35). The desired incoherent measurement on Alice's side now consists of the following incoherent Kraus operators: $K_{i}^{A}=|i\rangle\left\langle\left. e_{i}\right|^{A}\right.$. It can be verified by inspection that Bob's postmeasurement states indeed fulfill Eq. (35). This completes the proof of the lemma.

Lemma 4 implies that for any pure state $C_{\text {LICC }}^{A \mid B}$ is bounded below by the regularized coherence of assistance of Bob's reduced state:

$$
C_{a}^{\infty}\left(\rho^{B}\right) \leq C_{\mathrm{LICC}}^{A \mid B}\left(|\Psi\rangle^{A B}\right),
$$

where the regularized coherence of assistance is defined as [50] $C_{a}^{\infty}(\rho)=\lim _{n \rightarrow \infty} C_{a}\left(\rho^{\otimes n}\right) / n$. To prove this statement, consider the situation where Alice and Bob share $n \cdot m$ copies of the pure state $|\Psi\rangle=|\Psi\rangle^{A B}$. Using Lemma 4, it follows that in the limit of large $m$ Alice and Bob can use $n$ copies of $|\Psi\rangle$ to extract coherence at rate $C_{a}\left(\rho_{B}^{\otimes n}\right)$ on Bob's side, and thus

$$
C_{a}\left(\rho_{B}^{\otimes n}\right) \leq C_{\mathrm{LICC}}^{A \mid B}\left(|\Psi\rangle^{\otimes n}\right) .
$$

The proof of Eq. (37) is complete by dividing this inequality by $n$ and taking the limit $n \rightarrow \infty$. Equipped with these results, we are now in position to prove the following theorem. 
Theorem 5.-For any bipartite pure state $|\Psi\rangle=|\Psi\rangle\rangle^{A B}$ the following equality holds:

$$
\begin{aligned}
C_{\mathrm{LICC}}^{A \mid B}(|\Psi\rangle) & =C_{\mathrm{LQICC}}^{A \mid B}(|\Psi\rangle)=C_{\mathrm{SI}}^{A \mid B}(|\Psi\rangle) \\
& =C_{\mathrm{SQI}}^{A \mid B}(|\Psi\rangle)=C_{r}^{A \mid B}(|\Psi\rangle)=S\left(\Delta\left(\rho^{B}\right)\right) .
\end{aligned}
$$

Proof.-Combining Eqs. (20) and (37), we arrive at the inequality

$$
C_{a}^{\infty}\left(\rho^{B}\right) \leq C_{X}^{A \mid B}(|\Psi\rangle) \leq C_{r}^{A \mid B}(|\Psi\rangle)
$$

where $X$ is one of the sets considered above. The proof is complete by using the following equality, which holds for all pure states [50]:

$$
C_{r}^{A \mid B}(|\Psi\rangle)=C_{a}^{\infty}\left(\rho^{B}\right)=S\left(\Delta\left(\rho^{B}\right)\right)
$$

This result is surprising: it implies that the performance of the protocol does not depend on the particular set of operations performed by Alice and Bob. In particular, the optimal performance can already be reached by the weakest set of operations LICC, which restricts both Alice and Bob to local incoherent operations and classical communication. A better performance cannot be achieved if Alice is allowed to perform arbitrary quantum operations on her side (LQICC), and even if Alice and Bob have access to the most general set of operations considered here (SQI). This statement is true whenever Alice and Bob share a pure state.

\section{Maximally correlated states}

Here, we consider assisted coherence distillations for states of the form

$$
\rho^{A B}=\sum_{i, j} \rho_{i j}|i i\rangle\left\langle\left. j j\right|^{A B}\right.
$$

States of this form are known as maximally correlated states [60]. However, note that the family of states given in Eq. (42) does not contain all maximally correlated states, since $|i\rangle^{A}$ and $|j\rangle^{B}$ are incoherent states of Alice and Bob, respectively. We call these states maximally correlated in the incoherent basis. As we show in the following proposition, also for this family of states the inequalities Eq. (20) become equalities.

Proposition 6.-For any state $\rho=\rho^{A B}$ that is maximally correlated in the incoherent basis, the following equality holds:

$$
\begin{aligned}
C_{\mathrm{LICC}}^{A \mid B}(\rho) & =C_{\mathrm{LQICC}}^{A \mid B}(\rho)=C_{\mathrm{SI}}^{A \mid B}(\rho)=C_{\mathrm{SQI}}^{A \mid B}(\rho) \\
& =C_{r}^{A \mid B}(\rho)=S\left(\Delta^{B}(\rho)\right)-S(\rho) .
\end{aligned}
$$

Proof.-To prove this statement it is enough to prove the equality

$$
C_{\mathrm{LICC}}^{A \mid B}(\rho)=S\left(\Delta^{B}(\rho)\right)-S(\rho) .
$$

For this, we present a LICC protocol achieving the above rate. In particular, we show that there exists an incoherent measurement on Alice's side such that every postmeasurement state of Bob has coherence equal to $S\left(\Delta^{B}(\rho)\right)-S(\rho)$. The corresponding incoherent Kraus operators of Alice are given by $K_{j}^{A}=|j\rangle\left\langle\left.\psi_{j}\right|^{A}\right.$, where the states $\left.\mid \psi_{j}\right\rangle$ are mutually orthogonal, maximally coherent, and form a complete basis [61]. Since the states $\left|\psi_{j}\right\rangle$ are all maximally coherent, they can be written as $\left|\psi_{j}\right\rangle=1 / \sqrt{d_{A}} \sum_{k} e^{i \phi_{k}^{j}}|k\rangle$, with some phases $\phi_{k}^{j}$, and $d_{A}$ is the dimension of $A$. The corresponding postmeasurement states of Bob are then given by

$$
\rho_{j}^{B}=\sum_{k, l} e^{i\left(\phi_{l}^{j}-\phi_{k}^{j}\right)} \rho_{k l}|k\rangle\left\langle\left. l\right|^{B} .\right.
$$

If we now introduce the incoherent unitary $U_{j}=$ $\sum_{k} e^{i \phi_{k}^{j}}|k\rangle\langle k|$, we see that this unitary transforms the state $\rho_{j}^{B}$ to the state

$$
U_{j} \rho_{j}^{B} U_{j}^{\dagger}=\sum_{k, l} \rho_{k l}|k\rangle\left\langle\left. l\right|^{B} .\right.
$$

Since the relative entropy of coherence is invariant under incoherent unitaries, it follows that all states $\rho_{j}^{B}$ have the same relative entropy of coherence:

$$
C_{r}\left(\rho_{j}^{B}\right)=C_{r}\left(U_{j} \rho_{j}^{B} U_{j}^{\dagger}\right)=C_{r}\left(\sum_{k, l} \rho_{k l}|k\rangle\left\langle\left. l\right|^{B}\right) .\right.
$$

It is straightforward to verify that the right-hand side of this expression is equal to $S\left(\Delta^{B}(\rho)\right)-S(\rho)$, which completes the proof of the proposition.

The above proposition can also be generalized to states of the form

$$
\rho^{A B}=\sum_{i, j} \rho_{i j} U|i\rangle\left\langle\left. j\right|^{A} U^{\dagger} \otimes \mid i\right\rangle\left\langle\left. j\right|^{B},\right.
$$

where the unitary $U$ acts on the subsystem of Alice. In this case, the proposition can be proven in the same way, by applying the incoherent Kraus operators $K_{j}^{A}=|j\rangle\left\langle\left.\psi_{j}\right|^{A} U^{\dagger}\right.$ on Alice's side. 
These results show that the inequality Eq. (20) reduces to equality in a large number of scenarios, including all pure states, states that are maximally correlated in the incoherent basis, and even all states that are obtained from the latter class by applying local unitaries on Alice's subsystem. However, it remains open if Eq. (20) is a strict inequality for any mixed state.

\section{Remarks on the definition of $C_{X}$}

Here, we provide some remarks on the definition of $C_{X}$, where the set of operations $X$ is either LICC, LQICC, SI, or SQI. First, we note that the definition of $C_{X}$ given in Eq. (16) is equivalent to the following:

$C_{X}^{A \mid B}(\rho)=\sup \left\{R: \lim _{n \rightarrow \infty}\left(\inf _{\Lambda \in X}\left\|\operatorname{Tr}_{A}\left[\Lambda\left[\rho^{\otimes n}\right]\right]-\Psi_{2}^{\otimes[R n\rfloor}\right\|\right)=0\right\}$,

where $\lfloor x\rfloor$ is the largest integer below or equal to $x$ and $\Psi_{2}=\left|\Psi_{2}\right\rangle\left\langle\left.\Psi_{2}\right|^{B}\right.$ is a maximally coherent single-qubit state on Bob's side. To see that the expressions (16) and (49) are indeed equivalent, it is enough to note that every set of operations $X$ includes all incoherent operations on Bob's side, and that the theory of quantum coherence is asymptotically reversible for pure states; i.e., a state $\left|\psi_{1}\right\rangle$ with distillable coherence $c_{1}$ can be asymptotically converted into any other state $\left|\psi_{2}\right\rangle$ with distillable coherence $c_{2}$ at rate $c_{1} / c_{2}$ [39].

In the above discussion we implicitly assume that the Hilbert space of Alice and Bob has a fixed finite dimension, and that the incoherent operations performed by the parties preserve their dimension. One might wonder if the performance of any of the assisted distillation protocols $X$ discussed above changes if this assumption is relaxed, i.e., if Alice and Bob have access to additional local incoherent ancillas. This amounts to considering operations on the total state of the form $\rho^{A B} \otimes \sigma^{A^{\prime}} \otimes \sigma^{B^{\prime}}$, where

$$
\sigma^{A^{\prime}}=|0\rangle\left\langle\left. 0\right|^{A^{\prime}} \quad \text { and } \quad \sigma^{B^{\prime}}=\mid 0\right\rangle\left\langle\left. 0\right|^{B^{\prime}}\right.
$$

are additional incoherent states of Alice and Bob, respectively. As we see below, local incoherent ancillas cannot improve the performance of the procedure as long as SI and SQI operations are considered. For this, we first prove the following lemma.

Lemma 7.-For any SI operation $\tilde{\Lambda}_{\mathrm{SI}}$ acting on the state $\rho^{A B} \otimes \sigma^{A^{\prime}} \otimes \sigma^{B^{\prime}}$ there exists another SI operation $\Lambda_{\mathrm{SI}}$ acting on $\rho^{A B}$ such that the resulting state of $A B$ is the same:

$$
\Lambda_{\mathrm{SI}}\left[\rho^{A B}\right]=\operatorname{Tr}_{A^{\prime} B^{\prime}}\left[\tilde{\Lambda}_{\mathrm{SI}}\left[\rho^{A B} \otimes \sigma^{A^{\prime}} \otimes \sigma^{B^{\prime}}\right]\right]
$$

Proof.-This can be seen explicitly, by considering the form of a general SI operation $\tilde{\Lambda}_{\mathrm{SI}}$ acting on $\rho^{A B} \otimes$ $\sigma^{A^{\prime}} \otimes \sigma^{B^{\prime}}$ :

$$
\begin{aligned}
& \tilde{\Lambda}_{\mathrm{SI}}\left[\rho^{A B} \otimes \sigma^{A^{\prime}} \otimes \sigma^{B^{\prime}}\right] \\
& \quad=\sum_{i} \tilde{A}_{i} \otimes \tilde{B}_{i}\left(\rho^{A B} \otimes \sigma^{A^{\prime}} \otimes \sigma^{B^{\prime}}\right) \tilde{A}_{i}^{\dagger} \otimes \tilde{B}_{i}^{\dagger},
\end{aligned}
$$

where the operators $\tilde{A}_{i}$ and $\tilde{B}_{i}$ act on $A A^{\prime}$ and $B B^{\prime}$, respectively. The corresponding SI operation $\Lambda_{\mathrm{SI}}$ satisfying Eq. (51) is then given by

$$
\Lambda_{\mathrm{SI}}\left[\rho^{A B}\right]=\sum_{k, l, m} A_{k l m} \otimes B_{k l m}\left(\rho^{A B}\right) A_{k l m}^{\dagger} \otimes B_{k l m}^{\dagger} .
$$

The incoherent operators $A_{k l m}$ and $B_{k l m}$ depend on the operators $\tilde{A}_{i}$ and $\tilde{B}_{i}$ and have the following explicit form:

$$
\begin{aligned}
A_{k l m} & =\operatorname{Tr}_{A^{\prime}}\left[\tilde{A}_{k}\left(\mathbb{1}^{A} \otimes|0\rangle\left\langle\left. l\right|^{A^{\prime}}\right)\right],\right. \\
B_{k l m} & =\operatorname{Tr}_{B^{\prime}}\left[\tilde{B}_{k}\left(\mathbb{1}^{B} \otimes|0\rangle\left\langle\left. m\right|^{B^{\prime}}\right)\right],\right.
\end{aligned}
$$

where $\left\{|l\rangle^{A^{\prime}}\right\}$ is a complete set of incoherent states on $A^{\prime}$, and $\left\{|m\rangle^{B^{\prime}}\right\}$ is a complete set of incoherent states on $B^{\prime}$. Using the fact that $\tilde{A}_{k}$ and $\tilde{B}_{k}$ are incoherent, it is straightforward to verify that the operators $A_{k l m}$ and $B_{k l m}$ are also incoherent. Equation (51) can also be verified by inspection.

The above lemma implies that local incoherent ancillas on Alice's or Bob's side cannot improve the performance of the protocol if SI operations are considered. This can be seen by contradiction, assuming that by using a state $\rho^{A B}$ and local incoherent ancillas Bob can extract maximally coherent single-qubit states at rate $R>C_{\mathrm{SI}}^{A \mid B}$. Applying Theorem 2 and noting that the QI relative entropy does not change under attaching local incoherent ancillas, it follows that the rate $R$ is bounded above by $C_{r}^{A \mid B}$, which is again bounded above by $\log _{2} d_{B}$, where $d_{B}$ is the dimension of Bob's subsystem:

$$
\log _{2} d_{B} \geq C_{r}^{A \mid B}(\rho) \geq R>C_{\mathrm{SI}}^{A \mid B}(\rho) .
$$

The inequality $\log _{2} d_{B} \geq R$ means that if additional incoherent ancillas would improve the procedure at all, they are not needed at the end of the protocol and can be discarded [62]. These results together with Lemma 7 imply that the rate $R$ is also reachable without additional ancillas as long as SI operations are considered.

Very similar arguments can also be applied to the case of SQI operations. In this case, one can prove an equivalent statement to Lemma 7: for any SQI operation acting on $\rho^{A B} \otimes \sigma^{A^{\prime}} \otimes \sigma^{B^{\prime}}$ there exists a SQI operation acting on $\rho^{A B}$ such that the final state of $A B$ is the same. This implies that 
local incoherent ancillas cannot improve the performance in this case as well. It remains unclear if incoherent ancillas can provide advantage for LICC or LQICC operations. However, if Alice and Bob share a pure state, incoherent ancillas cannot provide any advantage also in this case due to Theorem 5.

\section{INCOHERENT TELEPORTATION}

In standard quantum teleportation introduced by Bennett et al. [63], Alice aims to transfer her single-qubit state to Bob by using LOCC together with one singlet. We now consider the task of incoherent teleportation, which is the same as standard teleportation up to the fact that LOCC is replaced by LICC. This means that Alice and Bob are allowed to apply only incoherent operations locally, and share their outcomes via a classical channel.

It seems that the restriction to local incoherent operations provides a severe constraint, and it is tempting to assume that Alice and Bob will not be able to achieve perfect teleportation in this way, at least if they have no access to additional coherent resource states. As we show in the following theorem, this intuition is not correct.

Theorem 8.-Perfect incoherent teleportation of an unknown state of one qubit is possible with one singlet and two bits of classical communication.

Proof.-To prove this statement, recall that in the standard teleportation protocol Alice applies a Bell measurement on her qubits $A$ and $A^{\prime}$ of the total initial state

$$
|\Psi\rangle=|\psi\rangle^{A^{\prime}} \otimes\left|\phi^{+}\right\rangle^{A B},
$$

where $\left|\phi^{+}\right\rangle^{A B}=(|00\rangle+|11\rangle) / \sqrt{2}$ is a maximally entangled state and $|\psi\rangle$ is the desired state subject to teleportation. Alice then communicates the outcome of her measurement to Bob. Depending on the outcome of Alice's measurement, either Bob finds his particle $B$ in the desired state $|\psi\rangle$ or he has to additionally apply one of the Pauli matrices $\sigma_{1}, i \sigma_{2}$, or $\sigma_{3}$.

It is now crucial to note that all Pauli matrices are incoherent: $\sigma_{i}|m\rangle \sim|n\rangle$. This means that Bob can perform his conditional rotation in an incoherent way. We now show that Alice's Bell measurement can also be performed in a locally incoherent way. For this, let $\left|\phi_{i}\right\rangle$ denote the four Bell states and consider the Kraus operators defined as $K_{i}=|00\rangle\left\langle\left.\phi_{i}\right|^{A A^{\prime}}\right.$. Note that these operators are local in Alice's lab, and, moreover, they are incoherent with respect to the bipartite incoherent basis of Alice. Finally, note that these Kraus operators lead to the same postmeasurement states of Bob as the projectors $\left|\phi_{i}\right\rangle\left\langle\left.\phi_{i}\right|^{A A^{\prime}}\right.$. This completes the proof of the theorem.

The above theorem shows that LICC operations are indeed powerful enough to allow for perfect teleportation. Since LICC is the weakest set of operations considered here, the same is also true for all the other sets LQICC, SI, and SQI: all these sets allow for perfect teleportation of an unknown qubit with one additional singlet. These results can be immediately extended to any system of $n$ qubits, in which case $n$ additional singlets are required.

\section{SUPERIORITY OF SQI OPERATIONS IN SINGLE-SHOT QUANTUM STATE MERGING}

In the discussion so far, and in particular in Eqs. (12), we see that the set SQI is strictly larger than LICC, LQICC, and SI. At this point it is important to note that a larger set of operations is not automatically more useful for real physical applications. Nevertheless, the results presented above indeed imply the existence of such physical tasks where the set SQI is more useful, when compared to the other sets individually. For completeness, we review these results in the following.

(i) SQI is superior to SI and LICC in the task of quantum state preparation. In particular, by starting from an initial state $|00\rangle^{A B}$, SQI operations can prepare all quantum-incoherent states, while only fully incoherent states can be prepared by SI and LICC operations; see Eqs. (10) and (11).

(ii) SQI is superior to LICC and LQICC in the task of quantum state discrimination. In particular, there exists a set of bipartite states that can be discriminated via SQI (and also via SI), but not via LICC and LQICC. This is discussed in detail in Sec. II; see Eq. (15).

It is now interesting to note that these two arguments are unrelated, and each of the arguments does not automatically imply the other one. In particular, the first argument for the superiority of SQI in comparison to SI and LICC cannot be used to show superiority in comparison to LQICC, since the set of states that can be prepared via SQI and LQICC is the same. On the other hand, the second argument showing superiority of SQI in comparison to LICC and LQICC cannot be used to show superiority in comparison to SI, since both SQI and SI are equally well suited for the considered task; see also Sec. II for more details. It is thus natural to ask for the existence of a quantum technological task that shows superiority of SQI operations with respect to all the other sets simultaneously.

In the following, we present such an application, which is based on the well-known task of quantum state merging. The latter task was introduced and studied in Refs. [47,48], and extended to the framework of coherence in Ref. [49]. In this task, three parties, Alice, Bob, and a referee, share a tripartite state $\rho^{R A B}$. The aim of the process is to send Bob's system to Alice [64] while keeping the overall state intact. In contrast to Refs. [47-49], we do not allow Alice and Bob to share any singlets, and, moreover, restrict them to the sets of operations considered in this paper, i.e., LICC, LQICC, SI, or SQI. 
We consider the merging of the following tripartite state:

$$
\rho^{R A B}=\frac{1}{9} \sum_{i}|i\rangle\left\langle\left. i\right|^{R} \otimes \mid \psi_{i}\right\rangle\left\langle\left.\psi_{i}\right|^{A B},\right.
$$

where $\left|\psi_{i}\right\rangle=\left|\alpha_{i}\right\rangle \otimes\left|\beta_{i}\right\rangle$ are nine $3 \times 3$ product states chosen as in Eq. (3) in Ref. [54] (see also the Appendix). Moreover, Alice has access to an additional register $A^{\prime}$ of dimension 3 in an incoherent initial state $|0\rangle^{A^{\prime}}$. Alice uses this register to store Bob's system. The total final state is given by

$$
\rho_{f}^{R A A^{\prime} B}=\Lambda_{X}\left[\rho^{R A B} \otimes|0\rangle\left\langle\left. 0\right|^{A^{\prime}}\right],\right.
$$

where $X$ denotes one of the four sets of operations we consider here. The process is successful if $\rho_{f}^{R A A^{\prime}}$ is the same as $\rho^{R A B}$ up to relabeling $B$ and $A^{\prime}$. Here, we consider the single-shot scenario; i.e., the corresponding operation is applied to one copy of the state only.

As we now show, this task can be performed via SQI, but not via any of the other three sets. For proving that the task can be performed via SQI, it is enough to recall that SQI operations can be used to distinguish the states $\left|\psi_{i}\right\rangle$. For each outcome $i$ Alice can then locally prepare her system $A A^{\prime}$ in the state $\left|\psi_{i}\right\rangle^{A A^{\prime}}$, which again can be achieved via SQI operations [65]. In the next step, we show that this task cannot be performed via LQICC operations. This can be seen by contradiction, assuming that LQICC operations can perform merging in this scenario, i.e., that

$\operatorname{Tr}_{B}\left[\Lambda_{\mathrm{LQICC}}\left[\rho^{R A B} \otimes|0\rangle\left\langle\left. 0\right|^{A^{\prime}}\right]\right]=\frac{1}{9} \sum_{i}|i\rangle\left\langle\left. i\right|^{R} \otimes \mid \psi_{i}\right\rangle\left\langle\left.\psi_{i}\right|^{A A^{\prime}}\right.\right.$,

for some LQICC protocol $\Lambda_{\mathrm{LQICC}}$. By linearity, it must be that

$$
\left|\psi_{i}\right\rangle\left\langle\left.\psi_{i}\right|^{A A^{\prime}}=\operatorname{Tr}_{B}\left[\Lambda_{\mathrm{LQICC}}\left[\left|\psi_{i}\right\rangle\left\langle\left.\psi_{i}\right|^{A B} \otimes \mid 0\right\rangle\left\langle\left. 0\right|^{A^{\prime}}\right]\right] ;\right.\right.
$$

i.e., Alice and Bob could use the protocol to transfer Bob's part of $\left|\psi_{i}\right\rangle^{A B}$ to Alice. Since the states $\left|\psi_{i}\right\rangle^{A B}$ form an orthonormal basis, this would imply that Alice and Bob could distinguish the states $\left|\psi_{i}\right\rangle^{A B}$ via LQICC (and thus also via LOCC), which is a contradiction to the main result of Ref. [54]. This also proves that the task cannot be performed via LICC operations.

It now remains to show that the task cannot be performed via SI operations. We prove this statement by using general properties of QI relative entropy given in Eq. (17) and its closed expression in Eq. (18). In particular, recall that the QI relative entropy cannot increase under SI operations, which implies that

$$
C_{r}^{R B \mid A A^{\prime}}\left(\rho_{f}^{R A A^{\prime} B}\right) \leq C_{r}^{R B \mid A A^{\prime}}\left(\rho^{R A B} \otimes|0\rangle\left\langle\left. 0\right|^{A^{\prime}}\right),\right.
$$

where $\rho_{f}$ is the final state given in Eq. (59). In the next step, we use the following relations:

$$
\begin{gathered}
C_{r}^{R \mid A A^{\prime}}\left(\rho_{f}^{R A A^{\prime}}\right) \leq C_{r}^{R B \mid A A^{\prime}}\left(\rho_{f}^{R A A^{\prime} B}\right), \\
C_{r}^{R B \mid A}\left(\rho^{R A B}\right)=C_{r}^{R B \mid A A^{\prime}}\left(\rho^{R A B} \otimes|0\rangle\left\langle\left. 0\right|^{A^{\prime}}\right),\right.
\end{gathered}
$$

which can be proven directly from the properties of QI relative entropy. Combining these results we arrive at the following inequality:

$$
C_{r}^{R \mid A A^{\prime}}\left(\rho_{f}^{R A A^{\prime}}\right) \leq C_{r}^{R B \mid A}\left(\rho^{R A B}\right) .
$$

In the final step of the proof, assume that SI operations allow us to perform the aforementioned task. We now show that this assumption leads to a contradiction. In particular, by our assumption the final state $\rho_{f}^{R A A^{\prime}}$ must be the same as $\rho^{R A B}$, up to relabeling $B$ and $A^{\prime}$. Thus, Eq. (65) is equivalent to

$$
C_{r}^{R \mid A B}\left(\rho^{R A B}\right) \leq C_{r}^{R B \mid A}\left(\rho^{R A B}\right) .
$$

By applying Eq. (18) together with the expression for the state $\rho^{R A B}$ in Eq. (58) and using the states $\left|\psi_{i}\right\rangle$ in Eq. (3) of Ref. [54] (see also the Appendix), we can now evaluate both sides of this inequality:

$$
\begin{aligned}
& C_{r}^{R \mid A B}\left(\rho^{R A B}\right)=\frac{8}{9}, \\
& C_{r}^{R B \mid A}\left(\rho^{R A B}\right)=\frac{4}{9},
\end{aligned}
$$

which is the desired contradiction. This finishes the proof that the task we consider here can be performed with SQI operations, but not with any other set LICC, LQICC, or SI.

Thus, we present the first example for a quantum technological application that can be performed via SQI operations, but cannot be performed with any of the other sets of operations considered in this paper.

\section{CONCLUSIONS}

In this paper, we study the resource theory of coherence in distributed scenarios. In particular, we focus on the following four classes of operations: local incoherent operations and classical communication (LICC), local quantum-incoherent operations and classical communication (LQICC), separable incoherent operations (SI), and separable quantum-incoherent operations (SQI). We show that these classes obey inclusion relations very similar to those between LOCC and separable operations known from entanglement theory.

We further study the role of these classes for the task of assisted coherence distillation, first introduced in Ref. [50]. 
Regardless of the particular class of operations, we prove that a bipartite state can be used for coherence extraction on Bob's side if and only if the state is not quantum incoherent. We also show that the relative entropy distance to the set of quantum-incoherent states provides an upper bound for coherence distillation on Bob's side, a result that again does not depend on the class of operations under scrutiny. Remarkably, both the SI and the SQI operations lead to the same performance in this task for all mixed states. For pure states an even stronger result is proven: in this case, all classes of operations we consider here are equivalent for assisted coherence distillation. We also study the task of incoherent teleportation, which arises from standard quantum teleportation by restricting the parties to LICC operations only. We show that in this situation LICC operations do not provide any restriction: perfect teleportation of an unknown qubit can be achieved with LICC and one additional singlet. Finally, we compare these classes on the task of single-shot quantum state merging. In this task, SQI operations provide an advantage with respect to all the other classes we consider here.

The tools we present here can be regarded as a first step towards a full resource theory of coherence in distributed scenarios. Indeed, while in the course of this work we focused on the coherence framework of Baumgratz et al. [5], it is important to note that the presented ideas are significantly more general. As an example, our tools can be directly applied to the situation where local incoherent operations are replaced by another well-justified set, such as strictly incoherent $[39,46]$, translationally invariant $[2,3,43,45]$, or physical incoherent operations [42]. These alternative frameworks have been extensively studied in recent literature, and each of them captures the concept of coherence in a different scenario [6]. Because of the close connection between all these frameworks, it is clear that the ideas we present in this work also carry over to these concepts. In particular, the results we present here, in general, serve as bounds for other concepts of coherence. We also expect that these bounds are tight in several relevant situations, e.g., for pure states. While the investigation of these questions is beyond the scope of this work, it has the potential to provide a unified view for all frameworks of coherence, and ultimately put the resource theory of coherence on equal footing with other quantum resource theories, most prominently the theory of entanglement [41].

Future research in this direction is also important in light of the recent progress towards understanding the role of coherence in quantum thermodynamics $[24,25]$. Here, the framework of thermal operations turned out to be very useful [66]. These operations arise from the first and second law of thermodynamics, and are known to be translationally invariant [24,25]. Because of this, the tools we develop in our work can also be applied to quantum thermodynamics. This research direction has the potential to reveal new surprising effects, similar to well-known phenomena such as bound entanglement $[67,68]$ in quantum information theory or the work-locking phenomenon [22,24,26,69] in quantum thermodynamics.

Recently, quantum coherence in multipartite systems has also been discussed by other authors [46,70,71]. While these works also study the role of coherence for quantum state manipulation, their motivation is significantly different from the concept we present here. In particular, the framework of coherence in multipartite systems is naturally suited for studying general nonclassical correlations such as quantum discord, as discussed in Refs. [46,70]. Another important research direction pursued in Refs. [70,71] is the role of coherence for deterministic quantum computation with one qubit (DQC1) [72]. This quantum protocol allows for efficient evaluation of the trace of a unitary, provided that the unitary has an efficient description in terms of twoqubit gates. Remarkably, this protocol does not require entanglement, while showing an exponential speed-up over the best known classical procedure [73]. The authors of Ref. [70] present a figure of merit for this task, which is related to the consumption of coherence in this protocol. Interestingly, while their figure of merit vanishes for unitaries of the form $U=e^{i \phi} \mathbb{1}$, it is unclear if a classical algorithm can evaluate the trace of this unitary efficiently [70]. In light of these results, we expect that the tools we present in our work can also find applications for the DQC1 protocol and quantum computation in general. This is beyond of the scope of the current work, and we leave it open for future research.

We also note that the class of LICC operations was introduced independently by Chitambar and Hsieh [74]. They study the tasks of asymptotic state creation and distillation of entanglement and coherence via LICC operations; i.e., local coherence is considered as an additional resource. They also independently obtain the results of our Theorems 3 and 5 and for LICC operations.

\section{ACKNOWLEDGMENTS}

We thank Gerardo Adesso, Eric Chitambar, Jens Eisert, Gilad Gour, Michał Horodecki, Paweł Horodecki, Iman Marvian, Martin B. Plenio, and Andreas Winter for discussion. We acknowledge financial supports from the Alexander von Humboldt-Foundation, the John Templeton Foundation, the EU grants OSYRIS (ERC-2013-AdG Grant No. 339106), QUIC (H2020-FETPROACT-2014 No. 641122), SIQS (FP7-ICT-2011-9 No. 600645), the Spanish MINECO grants (No. FIS2013-46768, No. FIS2008-01236, No. FIS2013-40627-P, and No. FIS2016-79508-P) with the support of FEDER funds and "Severo Ochoa" Programme (SEV-2015-0522), the Generalitat de Catalunya (2014-SGR-874, 2014-SGR-966, and CERCA Program), Fundació Privada Cellex, and the National Science Center in Poland (POLONEZ UMO2016/21/P/ST2/04054). 


\section{APPENDIX: THE NINE PRODUCT STATES FROM REF. [54]}

Here, we list the states $\left|\psi_{i}\right\rangle=\left|\alpha_{i}\right\rangle \otimes\left|\beta_{i}\right\rangle$ from Eq. (3) of Ref. [54]:

$$
\begin{aligned}
& \left|\psi_{1}\right\rangle=\left|\alpha_{1}\right\rangle \otimes\left|\beta_{1}\right\rangle=|1\rangle \otimes|1\rangle, \\
& \left|\psi_{2}\right\rangle=\left|\alpha_{2}\right\rangle \otimes\left|\beta_{2}\right\rangle=|0\rangle \otimes \frac{1}{\sqrt{2}}(|0\rangle+|1\rangle), \\
& \left|\psi_{3}\right\rangle=\left|\alpha_{3}\right\rangle \otimes\left|\beta_{3}\right\rangle=|0\rangle \otimes \frac{1}{\sqrt{2}}(|0\rangle-|1\rangle), \\
& \left|\psi_{4}\right\rangle=\left|\alpha_{4}\right\rangle \otimes\left|\beta_{4}\right\rangle=|2\rangle \otimes \frac{1}{\sqrt{2}}(|1\rangle+|2\rangle), \\
& \left|\psi_{5}\right\rangle=\left|\alpha_{5}\right\rangle \otimes\left|\beta_{5}\right\rangle=|2\rangle \otimes \frac{1}{\sqrt{2}}(|1\rangle-|2\rangle), \\
& \left|\psi_{6}\right\rangle=\left|\alpha_{6}\right\rangle \otimes\left|\beta_{6}\right\rangle=\frac{1}{\sqrt{2}}(|1\rangle+|2\rangle) \otimes|0\rangle, \\
& \left|\psi_{7}\right\rangle=\left|\alpha_{7}\right\rangle \otimes\left|\beta_{7}\right\rangle=\frac{1}{\sqrt{2}}(|1\rangle-|2\rangle) \otimes|0\rangle, \\
& \left|\psi_{8}\right\rangle=\left|\alpha_{8}\right\rangle \otimes\left|\beta_{8}\right\rangle=\frac{1}{\sqrt{2}}(|0\rangle+|1\rangle) \otimes|2\rangle, \\
& \left|\psi_{9}\right\rangle=\left|\alpha_{9}\right\rangle \otimes\left|\beta_{9}\right\rangle=\frac{1}{\sqrt{2}}(|0\rangle-|1\rangle) \otimes|2\rangle .
\end{aligned}
$$

[1] J. Åberg, Quantifying Superposition, arXiv:quant-ph/0612146.

[2] G. Gour and R. W. Spekkens, The Resource Theory of Quantum Reference Frames: Manipulations and Monotones, New J. Phys. 10, 033023 (2008).

[3] G. Gour, I. Marvian, and R. W. Spekkens, Measuring the Quality of a Quantum Reference Frame: The Relative Entropy of Frameness, Phys. Rev. A 80, 012307 (2009).

[4] F. Levi and F. Mintert, A Quantitative Theory of Coherent Delocalization, New J. Phys. 16, 033007 (2014).

[5] T. Baumgratz, M. Cramer, and M. B. Plenio, Quantifying Coherence, Phys. Rev. Lett. 113, 140401 (2014).

[6] A. Streltsov, G. Adesso, and M. B. Plenio, Quantum Coherence as a Resource, arXiv:1609.02439.

[7] D. Girolami, Observable Measure of Quantum Coherence in Finite Dimensional Systems, Phys. Rev. Lett. 113, 170401 (2014).

[8] D. Girolami and B. Yadin, Witnessing Multipartite Entanglement by Detecting Coherence, arXiv:1509.04131.

[9] X. Yuan, H. Zhou, Z. Cao, and X. Ma, Intrinsic Randomness as a Measure of Quantum Coherence, Phys. Rev. A 92, 022124 (2015).

[10] Y. Yao, X. Xiao, L. Ge, and C. P. Sun, Quantum Coherence in Multipartite Systems, Phys. Rev. A 92, 022112 (2015).

[11] X. Qi, Z. Bai, and S. Du, Coherence Convertibility for Mixed States, arXiv:1505.07387.

[12] A. Streltsov, U. Singh, H. Shekhar Dhar, M. Nath Bera, and G. Adesso, Measuring Quantum Coherence with Entanglement, Phys. Rev. Lett. 115, 020403 (2015).
[13] N. Killoran, F. E. S. Steinhoff, and M. B. Plenio, Converting Nonclassicality into Entanglement, Phys. Rev. Lett. 116, 080402 (2016).

[14] T. R. Bromley, M. Cianciaruso, and G. Adesso, Frozen Quantum Coherence, Phys. Rev. Lett. 114, 210401 (2015).

[15] U. Singh, M. Nath Bera, A. Misra, and A. Kumar Pati, Erasing Quantum Coherence: An Operational Approach, arXiv: 1506.08186.

[16] A. Mani and V. Karimipour, Cohering and Decohering Power of Quantum Channels, Phys. Rev. A 92, 032331 (2015).

[17] U. Singh, M. Nath Bera, H. Shekhar Dhar, and A. Kumar Pati, Maximally Coherent Mixed States: Complementarity between Maximal Coherence and Mixedness, Phys. Rev. A 91, 052115 (2015).

[18] T. Chanda and S. Bhattacharya, Delineating Incoherent Non-Markovian Dynamics Using Quantum Coherence, Ann. Phys. (Amsterdam) 366, 1 (2016).

[19] S. Lloyd, Quantum Coherence in Biological Systems, J. Phys. Conf. Ser. 302, 012037 (2011).

[20] C.-M. Li, N. Lambert, Y.-N. Chen, G.-Y. Chen, and F. Nori, Witnessing Quantum Coherence: From Solid-State to Biological Systems, Sci. Rep. 2, 885 (2012).

[21] S. F. Huelga and M.B. Plenio, Vibrations, Quanta and Biology, Contemp. Phys. 54, 181 (2013).

[22] P. Skrzypczyk, A. J. Short, and S. Popescu, Work Extraction and Thermodynamics for Individual Quantum Systems, Nat. Commun. 5, 4185 (2014).

[23] J. Åberg, Catalytic Coherence, Phys. Rev. Lett. 113, 150402 (2014).

[24] M. Lostaglio, D. Jennings, and T. Rudolph, Description of Quantum Coherence in Thermodynamic Processes Requires Constraints beyond Free Energy, Nat. Commun. 6, 6383 (2015).

[25] M. Lostaglio, K. Korzekwa, D. Jennings, and T. Rudolph, Quantum Coherence, Time-Translation Symmetry, and Thermodynamics, Phys. Rev. X 5, 021001 (2015).

[26] K. Korzekwa, M. Lostaglio, J. Oppenheim, and D. Jennings, The Extraction of Work from Quantum Coherence, New J. Phys. 18, 023045 (2016).

[27] G. Karpat, B. Çakmak, and F. F. Fanchini, Quantum Coherence and Uncertainty in the Anisotropic XY Chain, Phys. Rev. B 90, 104431 (2014).

[28] B. Çakmak, G. Karpat, and F. F. Fanchini, Factorization and Criticality in the Anisotropic XY Chain via Correlations, Entropy 17, 790 (2015).

[29] A. L. Malvezzi, G. Karpat, B. Çakmak, F. F. Fanchini, T. Debarba, and R. O. Vianna, Quantum Correlations and Coherence in Spin-1 Heisenberg Chains, Phys. Rev. B 93, 184428 (2016).

[30] J.-J. Chen, J. Cui, Y.-R. Zhang, and H. Fan, Coherence Susceptibility as a Probe of Quantum Phase Transitions, Phys. Rev. A 94, 022112 (2016).

[31] S. Cheng and M. J. W. Hall, Complementarity Relations for Quantum Coherence, Phys. Rev. A 92, 042101 (2015).

[32] X. Hu and H. Fan, Extracting Quantum Coherence via Steering, Sci. Rep. 6, 34380 (2016).

[33] D. Mondal, C. Datta, and S. Sazim, Quantum Coherence Sets the Quantum Speed Limit for Mixed States, Phys. Lett. A 380, 689 (2016). 
[34] P. Deb, Geometry of Quantum State Space and Quantum Correlations, Quantum Inf. Process. 15, 1629 (2016).

[35] S. Du, Z. Bai, and Y. Guo, Conditions for Coherence Transformations under Incoherent Operations, Phys. Rev. A 91, 052120 (2015).

[36] Z. Bai and S. Du, Maximally Coherent States, Quantum Inf. Comput. 15, 1355 (2015).

[37] M. N. Bera, T. Qureshi, M. A. Siddiqui, and A. K. Pati, Duality of Quantum Coherence and Path Distinguishability, Phys. Rev. A 92, 012118 (2015).

[38] X. Liu, Z. Tian, J. Wang, and J. Jing, Protecting Quantum Coherence of Two-Level Atoms from Vacuum Fluctuations of Electromagnetic Field, Ann. Phys. (Amsterdam) 366, 102 (2016).

[39] A. Winter and D. Yang, Operational Resource Theory of Coherence, Phys. Rev. Lett. 116, 120404 (2016).

[40] M. B. Plenio and S. Virmani, An Introduction to Entanglement Measures, Quantum Inf. Comput. 7, 1 (2007).

[41] R. Horodecki, P. Horodecki, M. Horodecki, and K. Horodecki, Quantum Entanglement, Rev. Mod. Phys. 81, 865 (2009).

[42] E. Chitambar and G. Gour, Critical Examination of Incoherent Operations and a Physically Consistent Resource Theory of Quantum Coherence, Phys. Rev. Lett. 117, 030401 (2016); see also, Comparison of Incoherent Operations and Measures of Coherence, Phys. Rev. A 94, 052336 (2016).

[43] I. Marvian and R. W. Spekkens, How to Quantify Coherence: Distinguishing Speakable and Unspeakable Notions, Phys. Rev. A 94, 052324 (2016).

[44] J. I. de Vicente and A. Streltsov, Genuine Quantum Coherence, J. Phys. A 50, 045301 (2017).

[45] I. Marvian, R. W. Spekkens, and P. Zanardi, Quantum Speed Limits, Coherence, and Asymmetry, Phys. Rev. A 93, 052331 (2016).

[46] B. Yadin, J. Ma, D. Girolami, M. Gu, and V. Vedral, Quantum Processes Which Do Not Use Coherence, Phys. Rev. X 6, 041028 (2016).

[47] M. Horodecki, J. Oppenheim, and A. Winter, Partial Quantum Information, Nature (London) 436, 673 (2005).

[48] M. Horodecki, J. Oppenheim, and A. Winter, Quantum State Merging and Negative Information, Commun. Math. Phys. 269, 107 (2007).

[49] A. Streltsov, E. Chitambar, S. Rana, M. N. Bera, A. Winter, and M. Lewenstein, Entanglement and Coherence in Quantum State Merging, Phys. Rev. Lett. 116, 240405 (2016).

[50] E. Chitambar, A. Streltsov, S. Rana, M. N. Bera, G. Adesso, and M. Lewenstein, Assisted Distillation of Quantum Coherence, Phys. Rev. Lett. 116, 070402 (2016).

[51] Eric M. Rains, Entanglement Purification via Separable Superoperators, arXiv:quant-ph/9707002.

[52] V. Vedral and M. B. Plenio, Entanglement Measures and Purification Procedures, Phys. Rev. A 57, 1619 (1998).

[53] E. Chitambar, D. Leung, L. Mančinska, M. Ozols, and A. Winter, Everything You Always Wanted to Know About LOCC (But Were Afraid to Ask), Commun. Math. Phys. 328, 303 (2014).

[54] C. H. Bennett, D. P. DiVincenzo, C. A. Fuchs, T. Mor, E. Rains, P. W. Shor, J. A. Smolin, and W. K. Wootters, Quantum Nonlocality without Entanglement, Phys. Rev. A 59, 1070 (1999).
[55] This should not be confused with strictly incoherent operations $[39,46]$.

[56] Note that the set of LOCC operations and the set of separable operations $\mathrm{S}$ cannot be included here, since they allow for local creation of coherence on Bob's side.

[57] H. Ollivier and W. H. Zurek, Quantum Discord: A Measure of the Quantumness of Correlations, Phys. Rev. Lett. 88, 017901 (2001).

[58] The QI relative entropy is continuous in the following sense [50]: for any two states $\rho=\rho^{X Y}$ and $\sigma=\sigma^{X Y}$ with $\|\rho-\sigma\| \leq 1, \quad$ it holds that $\left|C_{r}^{X \mid Y}(\rho)-C_{r}^{X \mid Y}(\sigma)\right| \leq$ $2 T \log _{2} d_{X Y}+2 h(T)$, where $T=\|\rho-\sigma\| / 2$ is the trace distance, $d_{X Y}$ is the total dimension, and $h(x)=-x \log _{2} x-$ $(1-x) \log _{2}(1-x)$ is the binary entropy.

[59] The states $\left|e_{i}\right\rangle$ are not necessarily incoherent.

[60] E. M. Rains, A Semidefinite Program for Distillable Entanglement, IEEE Trans. Inf. Theory 47, 2921 (2001).

[61] The existence of such states follows from the discrete Fourier transform.

[62] In particular, this means that Bob's local system $B$ is large enough to hold all maximally coherent states that can be potentially distilled via SI operations.

[63] C. H. Bennett, G. Brassard, C. Crépeau, R. Jozsa, A. Peres, and W. K. Wootters, Teleporting an Unknown Quantum State via Dual Classical and Einstein-Podolsky-Rosen Channels, Phys. Rev. Lett. 70, 1895 (1993).

[64] Note that in the standard approach [47-49] Alice sends her system to Bob. Here, we consider the other direction; i.e., Bob sends his system to Alice.

[65] The overall SQI Kraus operators for this procedure can be given as $K_{i j}^{A A^{\prime} B}=\left|\alpha_{i}\right\rangle\left\langle\left.\alpha_{i}\right|^{A} \otimes \mid \beta_{i}\right\rangle\left\langle\left. j\right|^{A^{\prime}} \otimes \mid 0\right\rangle\left\langle\left.\beta_{i}\right|^{B}\right.$.

[66] D. Janzing, P. Wocjan, R. Zeier, R. Geiss, and T. Beth, Thermodynamic Cost of Reliability and Low Temperatures: Tightening Landauer's Principle and the Second Law, Int. J. Theor. Phys. 39, 2717 (2000).

[67] P. Horodecki, Separability Criterion and Inseparable Mixed States with Positive Partial Transposition, Phys. Lett. A 232, 333 (1997).

[68] M. Horodecki, P. Horodecki, and R. Horodecki, MixedState Entanglement and Distillation: Is there a "Bound" Entanglement in Nature?, Phys. Rev. Lett. 80, 5239 (1998).

[69] M. Horodecki and J. Oppenheim, Fundamental Limitations for Quantum and Nanoscale Thermodynamics, Nat. Commun. 4, 2059 (2013).

[70] J. Ma, B. Yadin, D. Girolami, V. Vedral, and M. Gu, Converting Coherence to Quantum Correlations, Phys. Rev. Lett. 116, 160407 (2016).

[71] J. M. Matera, D. Egloff, N. Killoran, and M. B. Plenio, Coherent Control of Quantum Systems as a Resource Theory, Quantum Sci. Technol. 1, 01LT01 (2016).

[72] E. Knill and R. Laflamme, Power of One Bit of Quantum Information, Phys. Rev. Lett. 81, 5672 (1998).

[73] A. Datta, S. T. Flammia, and C. M. Caves, Entanglement and the Power of One Qubit, Phys. Rev. A 72, 042316 (2005).

[74] E. Chitambar and M.-H. Hsieh, Relating the Resource Theories of Entanglement and Quantum Coherence, Phys. Rev. Lett. 117, 020402 (2016). 